\title{
A 3-dimensional DTI MRI-based model of GBM growth and response to radiation therapy
}

\author{
LEITH HATHOUT $^{1}$, VISHAL PATEL $^{2}$ and PATRICK WEN ${ }^{1,3}$ \\ ${ }^{1}$ Harvard Medical School, Boston, MA 02115-5750; ${ }^{2}$ Department of Radiological Sciences, David Geffen School of Medicine, \\ UCLA, Los Angeles, CA 90024; ${ }^{3}$ Dana Farber Cancer Institute, Harvard University, Boston, MA 02115-5750, USA
}

Received April 6, 2016; Accepted May 23, 2016

DOI: 10.3892/ijo.2016.3595

\begin{abstract}
Glioblastoma (GBM) is both the most common and the most aggressive intra-axial brain tumor, with a notoriously poor prognosis. To improve this prognosis, it is necessary to understand the dynamics of GBM growth, response to treatment and recurrence. The present study presents a mathematical diffusion-proliferation model of GBM growth and response to radiation therapy based on diffusion tensor (DTI) MRI imaging. This represents an important advance because it allows 3-dimensional tumor modeling in the anatomical context of the brain. Specifically, tumor infiltration is guided by the direction of the white matter tracts along which glioma cells infiltrate. This provides the potential to model different tumor growth patterns based on location within the brain, and to simulate the tumor's response to different radiation therapy regimens. Tumor infiltration across the corpus callosum is simulated in biologically accurate time frames. The response to radiation therapy, including changes in cell density gradients and how these compare across different radiation fractionation protocols, can be rendered. Also, the model can estimate the amount of subthreshold tumor which has extended beyond the visible MR imaging margins. When combined with the ability of being able to estimate the biological parameters of invasiveness and proliferation of a particular GBM from serial MRI scans, it is shown that the model has potential to simulate realistic tumor growth, response and recurrence patterns in individual patients. To the best of our knowledge, this is the first presentation of a DTI-based GBM growth and radiation therapy treatment model.
\end{abstract}

\section{Introduction}

Glioblastoma (GBM) is both the most common and the most aggressive intra-axial brain tumor, with a notoriously poor prognosis $(1,2)$. Despite the aggressiveness of GBM, radiation

Correspondence to: Dr Leith Hathout, Harvard Medical School, 107 Avenue Louis Pasteur, Boston, MA 02115-5750, USA

E-mail: leith_hathout@hms.harvard.edu

Key words: GBM, modeling, diffusion tensor imaging, anisotropy therapy has been shown to significantly improve survival in multiple trials $(3,4)$. However, the standard approach to radiation therapy has remained relatively stable for several decades, using conformal radiation therapy with regimens such as $60 \mathrm{~Gy}$ over 30 doses of $2 \mathrm{~Gy}$ or $63 \mathrm{~Gy}$ over 35 doses of $1.8 \mathrm{~Gy}$ with $1-2 \mathrm{~cm}$ margins surrounding the visible tumor $(5,6)$.

These treatment plans constitute standardized therapeutic regimens which do not account for the biological heterogeneity of GBM among different patients (6). Thus, investigators have recently started trying to optimize radiation therapy protocols using a concept termed 'the fundamental principle of personalized management' (7). When applied to GBM, this concept seeks to individualize both prognostic estimates and treatment plans based on the biological parameters of each patient's tumor (6). It is suggested that this individualized approach may allow more tailored treatment based, for example, on differences in tumor invasiveness and proliferation, which would lead to different cell density profiles between patients $(6,8)$.

At the heart of these efforts is the push to develop accurate mathematical models of GBM growth and infiltration in the brain. Such models would not only have the potential to optimize therapy, but also to potentially predict individual patient response patterns to a proposed treatment regimen $(6,9,10)$.

Among the best known of these models is one originally pioneered by Murray et al (11), Harpold et al (12), Swanson et al (13) and Tracqui et al (14), using a reactiondiffusion partial differential equation model of cell density as a function of space and time. The model accounts for both the infiltrative nature of GBM using a diffusion term, and the net proliferation of tumor cells using a proliferation term (11-14).

The one-dimensional radially symmetric form of this model has been previously used to model the effects of both chemotherapy and surgery on the survival of patients with glioma (14,15). More recently, Rockne et al $(9,16)$ extended this model to include the effects of radiation therapy.

The one-dimensional model uses a single rate of diffusion, D, applicable throughout the brain, thereby modeling isotropic tumor growth $(12,16)$. However, such models do not simulate tumors in the context of actual brain anatomy, and in particular, ignore the differential infiltration of glioblastoma in the direction of white matter tracts. Thus, the most recent work has begun to incorporate diffusion tensor imaging (DTI) data into models of glioma growth, allowing for anisotropic tumor extension $(17,18)$. DTI-based growth models represent 
an important advance in tumor growth modeling because they allow 3-dimensional tumor modeling in the anatomical context of the brain. Specifically, tumor infiltration (modeled by the diffusion term) is guided by the direction of the white matter tracts along which glioma cells infiltrate. Such a 3D-DTI model was originally introduced by Jbabdi et al (17) to model the growth of low grade gliomas. In the present study, this model is applied to GBM, and extended to incorporate the effects of radiation therapy, thus producing a robust model which simulates both the growth of GBM and its response to radiation therapy. The model allows direct simulation of tumor growth with anisotropic infiltration along white matter tracts, along with estimates of cell density gradients. It has the potential to model different tumor growth patterns based on location within the brain, and response patterns to different radiation therapy regimens. When combined with the potential of being able to estimate the biological parameters of invasiveness and proliferation from serial MRI scans for a specific patient, it is hoped that such a model will represent a significant advance in individualized treatment regimens and prognostic estimates.

To the best of our knowledge, this is the first presentation of a DTI-based GBM growth and radiation therapy treatment model. Using the model, a GBM is 'grown' in brain tissue, then treated with radiation, and then monitored as it naturally recurs via the model.

\section{Materials and methods}

The 3D-DTI model. In its initial formulation, the reactiondiffusion model was used to simulate glioblastoma growth in one spatial dimension:

$$
\frac{\partial c}{d t}=D \frac{\partial^{2} c}{d x^{2}}+\rho c\left(1-\frac{c}{K}\right)
$$

where the various terms are defined as follows: $c(t, x)$ is the tumor cell density, in terms of cells $/ \mathrm{mm}^{3}$, which is a function of position $x$ and time $t$.

$\mathrm{D}(x)$ is the diffusion term, in $\mathrm{mm}^{2} /$ day, which models local tumor invasion of tumor cells.

$$
\rho c\left(1-\frac{c}{K}\right)
$$

is a logistic tumor growth term, where $\rho$ is the tumor proliferation rate in units of (/day), governed by a tissue tumor carrying capacity $\mathrm{K}$, in units of cells $/ \mathrm{mm}^{3}$.

With the 3D-DTI model, D becomes a tensor incorporating the magnitude and directionality of the diffusion coefficient (17).

Thus, the model can be rewritten as:

$$
\frac{\partial c(t, x)}{d t}=\nabla \bullet(\bar{D}(x) \nabla c)+\rho c\left(1-\frac{c}{K}\right)
$$

where $\bar{D}(x)$ is the $3 \times 3$ diffusion tensor that describes anisotropic diffusion as a function of location. This diffusion tensor is derived from the standard water diffusion tensor

$$
\bar{D}_{w}=\left(\begin{array}{ccc}
D_{x x} & D_{x y} & D_{x z} \\
D_{y x} & D_{y y} & D_{y z} \\
D_{z x} & D_{z y} & D_{z z}
\end{array}\right) .
$$

$\bar{D}_{w}$ is then modified by the use of a 'stretch factor' as per Jbabdi et al (17) to account for the fact that cellular migration along white matter tracts displays greater anisotropy than does water diffusion.

After $\bar{D}(x)$ has been calculated, the equation can be developed as follows: once again as per Jbabdi et al (17),

$$
\frac{\partial c(t, x)}{d t}=\nabla \bullet(\bar{D}(x) \nabla c)+\rho c\left(1-\frac{c}{K}\right)
$$

can be transformed into the following equation:

$$
\frac{\partial c(t, x)}{d t}=\sum_{i, j=1}^{3} \bar{D}_{i, j} \frac{\partial^{2} c}{\partial x_{i} \partial x_{j}}+\sum_{i=1}^{3} \widetilde{\bar{D}}_{i} \frac{\partial c}{\partial x_{i}}+\rho c\left(1-\frac{c}{K}\right)
$$

where

$$
\widetilde{\bar{D}}_{i}=(\bar{D} \nabla)_{i}=\sum_{j=1}^{3} \frac{\partial \bar{D}_{i, j}}{\partial x_{j}} .
$$

For any given initial condition $c(0, x)=c_{o}(x)$ and zero flux boundary conditions such that tumor cells are prohibited from migrating outside the domain of the brain or into the ventricles $(\bar{D}(x) \nabla c) \cdot \vec{n}=0$, this model can be used to evaluate tumor cell concentration as a function of both time and space.

The model is then solved using a forward finite differences approach, using $\Delta t$ as a time step and $(\Delta x, \Delta y, \Delta z)$ as space steps (17).

The initial condition used in the present study is a tumor cell concentration of $0.8 * \mathrm{~K}$ confined to a single voxel.

$\mathrm{K}$ is the tissue tumor carrying capacity in units of cells $/ \mathrm{mm}^{3}$ and is typically set at $10^{5} \mathrm{cell} / \mathrm{mm}^{3}$ (19).

The trace of the diffusion tensor is scaled to a global D value which is similar to the one-dimensional model. For the current model, various values of $\mathrm{D}$ and $\rho$ are used for simulations, drawn from the range of published values for GBM $(16,20)$.

The model predicts a gradient of cell density, gradually advancing from the center of the tumor guided by the diffusion coefficient and the direction of adjacent white matter tracts. The model then uses cell density thresholds for tumor visibility on both $\mathrm{T} 1$ post contrast images and FLAIR/T2 images. The $\mathrm{T} 1$ post contrast detection threshold, used to simulate the enhancing $\mathrm{T} 1$ tumor radius (T1C), is set at $0.8 \mathrm{~K}$, while the T2 threshold is $0.16 \mathrm{~K}$, keeping consistent with estimates from prior work (6).

Radiation therapy. To model treatment, an additional radiation therapy term is added:

$$
\frac{\partial c(t, x)}{d t}=\nabla \bullet(\bar{D}(x) \nabla c)+\rho c\left(1-\frac{c}{K}\right)-R(S, c(x, t))
$$

Similar to the work of Rockne et al $(9,10)$, the R term quantifies the loss of tumor cells due to radiation therapy, which is delivered as discrete doses, and is hence amenable to modeling different dosing schedules. $\mathrm{R}$ is defined as a function of $\mathrm{S}$, the fraction of cells surviving a radiation dose, using the wellknown linear-quadratic dose-response model: $S=e^{-(\alpha d+\beta d 2)}$, where $\alpha$ (in units of $\mathrm{Gy}^{-1}$ ) and $\beta$ (in units of $\mathrm{Gy}^{-2}$ ) reflect type A (single ionizing event) and type B (pairwise interaction of ionizing events) tissue damage. Since tissues can be somewhat characterized by an $\alpha / \beta$ ratio, and to simplify the model to a single radiation parameter, the $\alpha / \beta$ is held fixed, as in prior 
work $(6,9,10)$. In the present study, similar to prior studies, this ratio is held at 10 Gy $(6,9,10)$. Thus, $\mathrm{S}$ can be written as:

$$
S=e^{-\alpha\left(d(x, t)+\frac{d^{2}(x, t)}{\alpha / \beta}\right)}
$$

It is noted that this formulation is fully in accordance with the standard definition of B.E.D., the biologically effective radiation dose, which is defined as:

$$
\text { B.E.D. }=d\left(1+\frac{d}{\alpha / \beta}\right)
$$

Hence, $\mathrm{S}$ can be rewritten as $S=e^{-\alpha(B . E \cdot D)}(16)$.

If $S$ is the surviving cell fraction for a given dose, then (1-S) is the probability of cell death. In this formulation, $\alpha$ can be regarded as the parameter defining radiation sensitivity. A higher $\alpha$ corresponds to a decreased probability of survival. Also $d$ reflects the given radiation dose, which is a function of both space and time, $d(x, t)$, allowing specific radiation therapy protocols to be modeled.

As per Rockne et al $(9,10)$, the effect of radiation therapy is also cast as a function of cell density, using the same logistic formulation as the tumor growth model as follows:

$$
R=(1-S) \cdot c\left(1-\frac{c}{K}\right)
$$

Hence, at locations of high cell density, when the tumor cell proliferation is decreased according to the logistic configuration, the effect of radiation therapy is likewise decreased. This is consistent with the understanding that radiation is most effective in regions of high mitotic activity and high cellular turnover. Conversely, at low cell densities, the effect of radiation therapy is essentially linearly related to the fraction of cells killed. The above formulation is equivalent to a Taylor series approximation of having the logistic term as part of the exponent of $\mathrm{S}$.

In keeping with previous studies, radiation therapy is modeled as a total of $61.2 \mathrm{~Gy} / 34$ doses, using the University of Washington protocol (16). The first 28 doses are given in a field using the T2 tumor boundary $+2.5 \mathrm{~cm}$, while the last 6 doses are given as a more spatially limited booster field using the enhanced $\mathrm{T} 1$ radius $+2 \mathrm{~cm}$.

Relying on previously published estimates, including the variation of $\alpha$ with tumor proliferation, various values of are used, ranging from an $\alpha$ of $0.03 \mathrm{~Gy}^{-1}$ to an $\alpha$ of $0.09 \mathrm{~Gy}^{-1}(16,21)$.

MR imaging. The model simulation was carried out on a DTI series from a healthy volunteer. Imaging was performed on a Siemens 3T Magnetom scanner, with standard T1 (TR 487 ms, TE $16 \mathrm{~ms}, 5 \mathrm{~mm}$ slice thickness) and T2 (TR $3670 \mathrm{~ms}$, TE $93 \mathrm{~ms}, 5 \mathrm{~mm}$ slice thickness). DTI imaging was performed using a 128 x 128 matrix with a voxel size of 1.7 x 1.7 x $4 \mathrm{~mm}$, TR $4100 \mathrm{~ms}$, TE $95 \mathrm{~ms}$, using a diffusion schema with 64 non-colinear imaging directions, b-values $=0$ and $1000 \mathrm{~s} / \mathrm{mm}^{2}$, $\mathrm{NEX}=3$, with 30 contiguous slices covering the entire brain.

\section{Results}

The model can be used to simulate GBM growth, showing a realistic growth pattern, with tumor spreading along the direction of fiber tracts as mapped by DTI (Figs. 1 and 2).
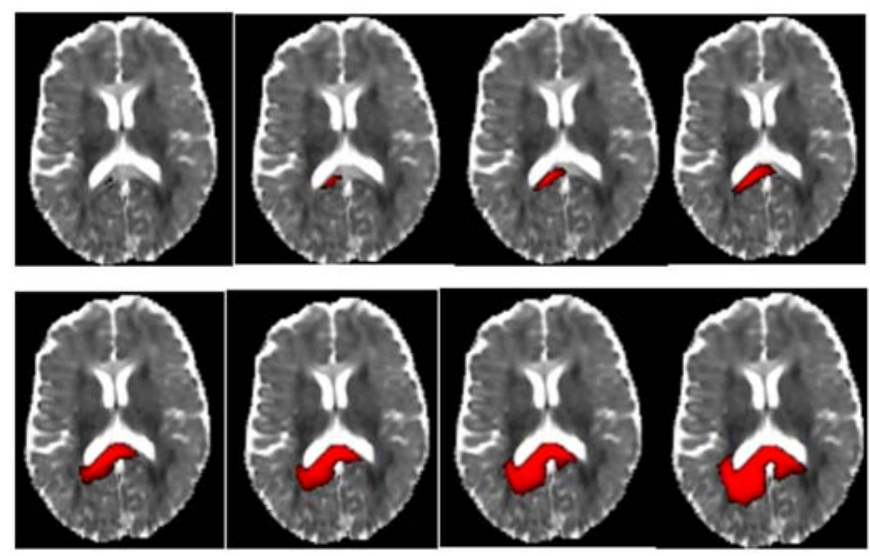

Figure 1. Serial axial slices of a simulated tumor seeded in the right aspect of the splenium of the corpus callosum, overlaid on a DTI image, with $\mathrm{D}=0.04 \mathrm{~mm}^{2} /$ day and $\rho=0.05 /$ day. Images are at $105,135,165,195,240,270$ and 345 days. The model shows tumor growing along adjacent fiber tracts.

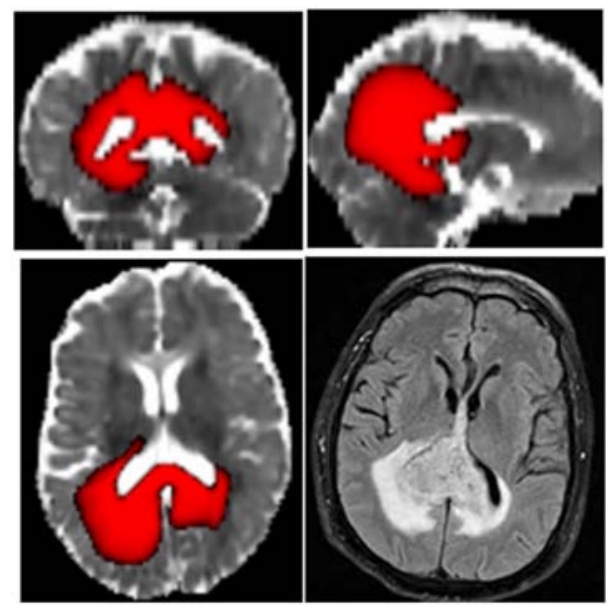

Figure 2. (Top left to bottom right) Coronal, sagittal and axial slices of the simulated tumor from above at 405 days. An axial view of an actual GBM in the corpus callosum is shown for comparison. The DTI growth model provides a fairly accurate simulation of tumor growth. GBM image from Kiely and Twomey (31).

The model can also be used to simulate the response to radiation therapy, as per the Materials and methods. There is visible tumor shrinkage with radiation therapy, and this is a function of both the tumor parameters D and $\rho$, as well as the radiation sensitivity as set by $\alpha$. In Fig. 3, a simulation of tumor response to a standard radiation therapy protocol (Materials and methods) is shown for two tumors. By setting different cell density thresholds, the model can also simulate what is referred to as the 'subthreshold zone', of low density tumor cells that are below the T2 visibility threshold. Although the second tumor ( $\mathrm{D}=0.4 \mathrm{~mm}^{2} /$ day and $\rho=0.04 /$ day) shows a more pronounced response to radiation therapy on T1C images (Fig. 3B vs. E), the model suggests that after treatment, the second tumor actually has a larger subthreshold zone of low density infiltration of tumor cells streaming through the white matter tracts into adjacent brain, including crossing the splenium of the corpus callosum. This is because the second tumor has a higher diffusion coefficient ( $D=0.4 \mathrm{~mm}^{2} /$ day vs. $0.2 \mathrm{~mm}^{2} /$ day). Thus, the 

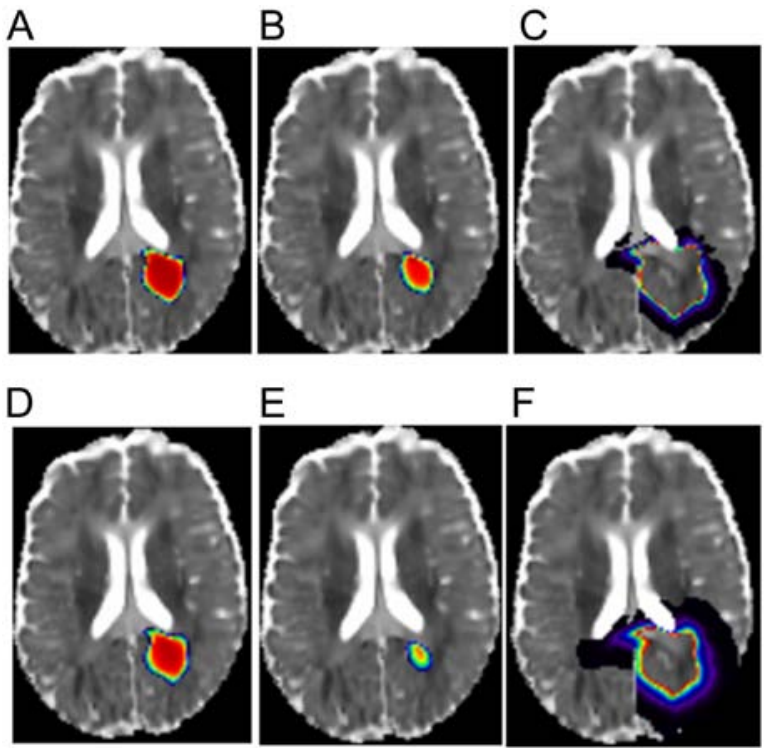

Figure 3. Simulations of the response of two tumors to radiation therapy. (A-C) Are for a tumor with $\mathrm{D}=0.2 \mathrm{~mm}^{2} /$ day and $\rho=0.1 /$ day, representing a fairly aggressive GBM. For this tumor, is set at $0.09 \mathrm{~Gy}^{-1}$. (D-F) Are for a tumor with $\mathrm{D}=0.4 \mathrm{~mm}^{2} /$ day and $\rho=0.04 /$ day. For this tumor, $\alpha$ is set at $0.06 \mathrm{~Gy}^{-1}$. (A and D) Projected T1C appearance of tumor prior to radiation therapy. (B and E) Projected T1C appearance after radiation therapy. (C and F) Simulated post-therapy subthreshold zone appearance of tumor, with a projection of tumor extension beyond the visible T2 margins. For this and all figures, red represents the highest cellular concentrations, while yellow, violet, blue and black represent progressively lower concentrations.

model gives some insight into the complex nature of tumor growth and treatment.

The model also allows the simulation of any desired radiation therapy protocol, and hence provides a simulation environment within the anatomic context of the brain where different therapeutic protocols may be experimented with, at least to examine qualitative differences. For example, in Fig. 4, the standard radiation therapy protocol is compared to a hypofractionation protocol. Hypofractionation protocols similar to the one below have been tried in some studies with a thought to limiting the number of hospital visits which patients with a limited life expectancy need to make for treatment, and hopefully improving quality of remaining life (22).

The most significant potential use of the model, however, would be to model a priori the expected growth pattern and response to treatment in a given patient. This will require a significant amount of correlation between the model and actual patient data, as well as how to 'calibrate' the model for an individual patient's GBM beyond location of the tumor. However, Fig. 5 is presented to illustrate the potential of the model to simulate tumor response to therapy and tumor recurrence post therapy. The comparison of simulation to actual images shows a reasonably accurate set of results both in terms of tumor morphology and in terms of time frame. The model images were obtained at time-points matching the available MRI scans and dates of radiation therapy.

\section{Discussion}

Accurate mathematical modeling of glioblastoma growth and spread is an important adjunct to developing both optimal treatment plans and accurate prognostic estimates. The reaction-diffusion partial differential equation model provides a method of doing this by characterizing both the infiltration and proliferation of glioblastoma $(11,12)$.

In general terms, this model has been used to simulate the response of GBM to both surgery and chemotherapy $(14,15)$ as a function of the model's parameters. More importantly, the model has shown the capacity to characterize the behavior of GBM in individual patients. This is done by estimating the tumor parameters, D and $\rho$, from two serial MR scans (6). In this way, the model has been used to estimate the survival of individual patients as well as individual response to radiation therapy $(9,13)$.

In this initial form, the reaction-diffusion equation was used to model isotropic diffusion of tumor cells in one spatial dimension. In such a context, there is a single, constant value of $\mathrm{D}$ that is applicable throughout the domain of the brain $(11,16)$. However, this assumption does not capture the anatomical complexities of the brain. Hence, based on the finding that glioma cells diffuse more quickly through white matter than gray matter, a refinement of the model by Swanson et al incorporated preferential white matter diffusion into the reaction-diffusion tumor model, with D now taking different values in gray matter $\left(D_{g}\right)$ and white matter $\left(D_{w}\right)(12,23)$. However, even in white matter, it is known that diffusion is not isotropic, and that tumor cells migrate preferentially in the direction of white matter tracts (24-28). Thus, the most recent research has begun to incorporate diffusion tensor MR imaging (DTI) data

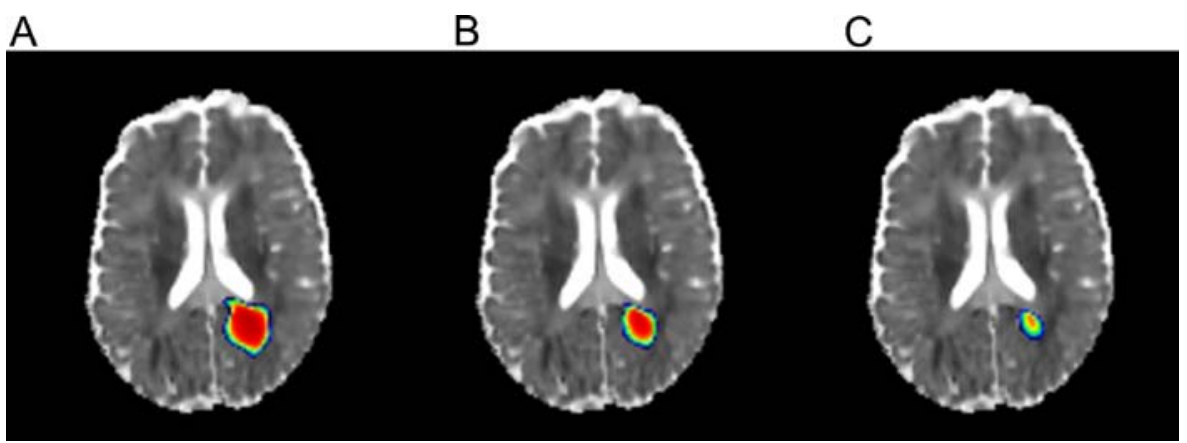

Figure 4. Simulations of the effects of different radiation therapy protocols. Simulation of a tumor with $\mathrm{D}=0.4 \mathrm{~mm} / \mathrm{day}$ and $\rho=0.04 / \mathrm{day}, \mathrm{with} \alpha=0.06 \mathrm{~Gy}{ }^{-1}$. (A) Pretherapy T1C. (B) Post-therapy T1C with an accelerated hypofractionated protocol consisting of 35 Gy given as 5 doses of 7 Gy/dose, one dose per week. (C) Post-therapy T1C with standard radiation protocol described in Materials and methods. 

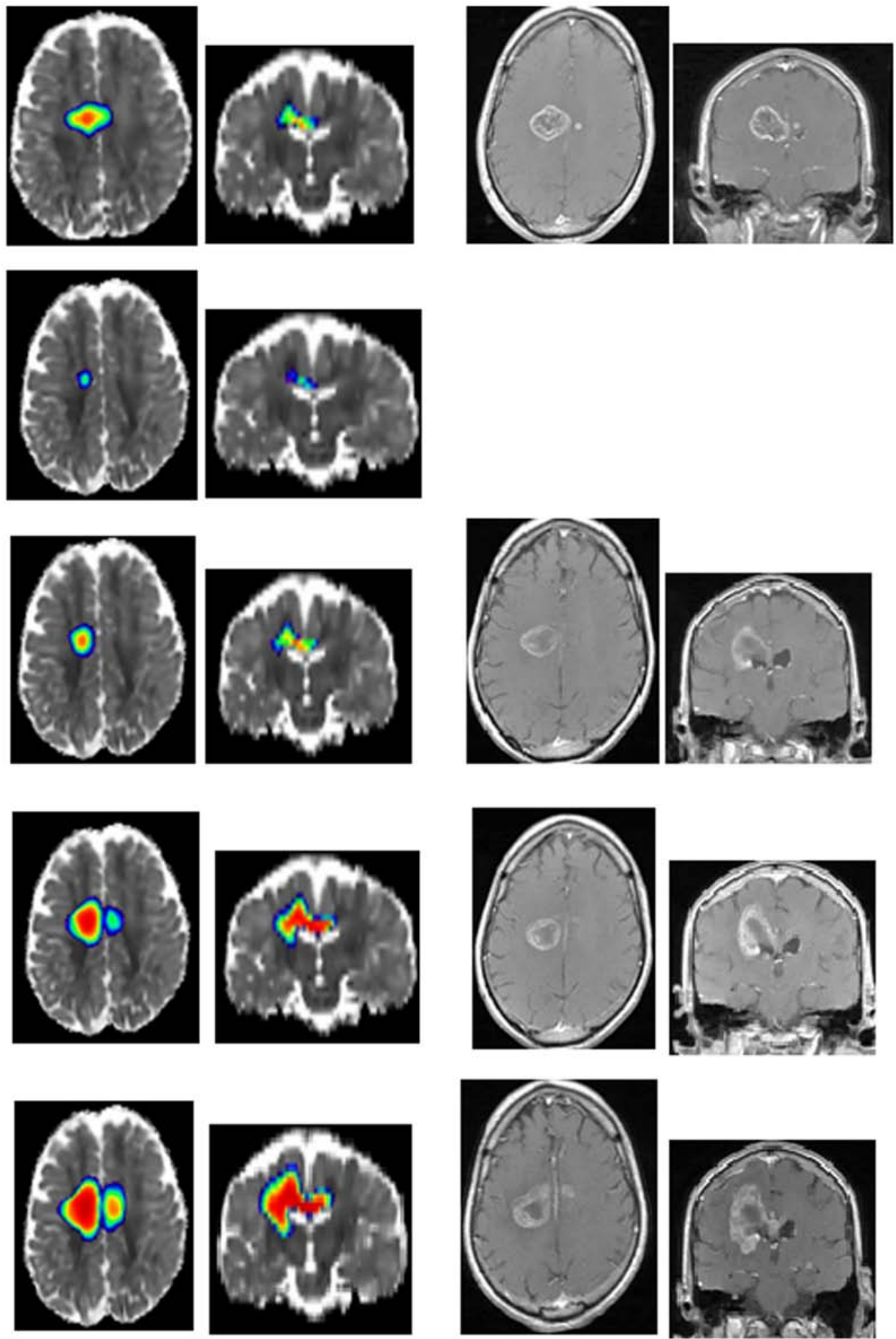

Figure 5. Model simulations of an individual GBM patient who underwent only radiation therapy. Row 1, day 0, when patient presented; simulated T1C vs. actual T1C axial and coronal images. The simulated and actual tumors have a similar shape in the centrm semiovale, slightly ovoid with a horizontal long axis, but with central anterior and posterior protrusions. Patient underwent radiation therapy on day 12, with 60 Gy over 30 doses in 6 weeks. Row 2 , simulated response to radiation therapy. No MR images available immediately post-therapy. Row 3, day 102 . Axial and coronal simulated and actual MR images. Tumor has begun to regrow post-therapy. However, simulations show decreased intra-tumoral cell concentrations compared to pre-therapy images, corresponding to less internal enhancement in tumor on actual MR images. Row 4, day 146, actual vs. simulated images, with increased tumor growth and spread across the corpus callosum. Row 5, day 177, actual vs. simulated tumor, with further tumor growth including further spread across the midline. Both the simulated and actual coronal images show tumor wrapping around the inferolateral margin of the right lateral ventricle in very similar configurations.

into models of glioma growth, allowing for modeling anisotropic tumor growth $(17,18)$. In this latest refinement of the model, tumor infiltration (modeled by the diffusion term) is guided by the direction of the white matter tracts along which glioma cells infiltrate, based on a diffusion tensor associated with each voxel (17). This study implements the 3d-DTI model 
and further refines it by incorporating radiotherapy into the model. This model thus provides the most realistic available tool for modeling GBM growth and response to therapy. In Fig. 1, the model simulates the growth of a butterfly glioma beginning at the right margin of the splenium of the corpus callosum. It is noted that the pattern of growth is anatomically realistic, and also provides reasonable temporal estimates of tumor growth. The evolution of the GBM and the distribution of tumor cells in the brain are thus seen to be entirely different in this model than would be predicted by an isotropic spherically symmetric model. Furthermore, by incorporating volumetric DTI data, the model allows a true 3-dimensional simulation of tumor growth within the brain volume (Fig. 2), rather than the concatenation of 2-dimensional slices to create a 3-dimensional volume.

The new model presented here also has the capacity to simulate response to radiation therapy. In Fig. 3, the response of two different tumors is compared. In the first tumor, $\mathrm{D}=0.2 \mathrm{~mm}^{2} /$ day and $\rho=0.1 /$ day. This tumor, because of its relatively high proliferation coefficient, shows a positive but fairly limited response to radiation therapy. In the second tumor, $\mathrm{D}=0.4 \mathrm{~mm}^{2} /$ day and $\rho=0.04 /$ day. In this tumor, there is significantly more shrinkage with radiation therapy, as well as decreased cellular concentrations within the remaining tumor. Most interestingly, the current model also allows estimation of the subthreshold zone of 'invisible' tumor cells, below the T2 visibility threshold (Fig. 3C and F). It is noted that although the second tumor shows a significantly better response to radiation therapy on T1C, it actually has a larger zone of subthreshold tumor cells spreading beyond the radiation field, because of a higher diffusion coefficient. Of particular interest is the spread of a low concentration of tumor cells to the contralateral hemisphere across the splenium of the corpus callosum (Fig. 3F). The extent and pattern of the subthreshold zone are of significant import in planning and optimizing radiation fields (18).

The model thus provides a virtual in silica laboratory to compare the expected response to different radiation therapy protocols. In Fig. 4, the same tumor is treated with the traditional protocol (see Materials and methods), as well as with a hypofractionated protocol, with a total dose of 35 Gy delivered in 5 doses of $7 \mathrm{~Gy}$, one dose per week. The model predicts a positive response to both therapies, but with a better response to the traditional dosing regimen. These results are in keeping with some of the empirical results on hypofractionated therapy. For example, the work of McAleese et al (29) compared an accelerated hypofractionated protocol consisting of $30 \mathrm{~Gy}$ in 6 fractions to matched controls receiving a standard $60 \mathrm{~Gy} / 30$ fraction protocol, and found that although the hypofractionated protocol had a palliative benefit, it showed a shorter median survival of 2.5-4.5 months compared to the standard protocol. Of course, the objective of the present study is not to rigorously compare hypofractionated to standard protocols, but the simulation is presented to show the utility of the current model.

Most importantly, the model can also potentially be used to simulate the growth of GBM and its response to treatment in individual patients, as seen in Fig. 5. That figure illustrates the case of a patient treated only with a standard radiation therapy protocol, and compares the results of the model to the patient's images. The initial simulation images, with a tumor grown in the right centrum semiovale, show a tumor shape similar to the actual images, with a tumor that is slightly ovoid, with a horizontal long axis, but with small anterior and posterior protuberances, and with a slight extension across the midline (Fig. 5, first row). The time course and growth pattern of the tumor recurrence after radiation therapy are comparable to the patient's follow-up images. The coronal image growth patterns across the corpus callosum and the extension around the frontal horn of the right lateral ventricle are quite similar. Most interestingly, there is a change in tumor shape after radiation therapy, with a shift from an ovoid shape with a horizontal long axis to a rounded shape and then to tumor growth with a more anterior-posterior long axis (Fig. 5). Of course, this case represents only anecdotal evidence of the utility of the model, and is once again presented simply to illustrate the potential utility of the model.

The present study thus presents a significant refinement to existing GBM growth and treatment models, and initial investigations suggest that the model's results are at least a reasonable facsimile to reality. The model provides a method of predicting the spread of GBM along white matter tracts, and provides estimates of the extent of 'subthreshold' tumor below the MR visibility threshold (Fig. 3C and F). Also, the model provides a method to simulate the response of GBM to different radiation therapy regimens, as in Fig. 4, where the model predicts that the standard protocol is more effective than the modeled hypofractionation protocol. Notably, when the same comparison is carried out for a tumor with a $\rho$ of $0.1 /$ day (results not shown), the model predicts that the advantage of the standard protocol becomes more pronounced as the proliferation index of the tumor increases.

All of these results are preliminary, and the utility of the model will only be elucidated by close empirical comparisons in future studies. Of course, the current model still has limitations. For example, it is unclear whether it is sufficient to model GBM invasiveness as a passive diffusion process. Also, to individualize the model, the D and $\rho$ of the tumor need to be determined, which requires two serial MR scans, and this is quite uncommon for GBM. Therefore, the model may need to rely on average values and provide only qualitative predictions. Much empirical testing will need to be performed to see if the model will be quantitatively accurate in terms of the growth of GBM and its response to therapy even if values of $\mathrm{D}$ and $\rho$ are known. The radiation therapy simulations also rely on the linear-quadratic model, and on values of $\alpha$ and a $\alpha / \beta$ fixed ratio, which are only rough approximations. Initial results, for example, suggest that the value of $\alpha$ varies with the proliferative index of the tumor (9). Also, temozolomide has become a standard part of glioblastoma treatment, and it remains unclear how to incorporate the effects of temozolomide into the model. Initial results by Barazzuol et al (21) suggests that temozolomide enhances radiation sensitivity, and that this can be modeled as a decrease in the $\alpha / \beta$ ratio. If so, that can be easily incorporated into the model, but again will require close correlation to empirical data. That said, evidence suggests that temozolomide is less effective for the subset of GBM with unmethylated MGMT promoter, and thus the impact of excluding temozolomide from the current treatment model is likely to be smaller for this subset (30). The current model focuses on radiation therapy, and does 
not include surgery simulations. Once again, this can also be incorporated into future iterations of the model, and will be necessary for accurate modeling of tumor treatment and recurrence. However, even with these significant limitations, it is hoped that the current model provides an important addition to current modeling efforts, and shows encouraging initial results and insights into the morphology of GBM growth, spread, response to therapy and recurrence after treatment.

\section{References}

1. Ostrom QT, Gittleman H, Fulop J, Liu M, Blanda R, Kromer C, Wolinsky Y, Kruchko C and Barnholtz-Sloan JS: CBTRUS Statistical Report: Primary Brain and Central Nervous System Tumors Diagnosed in the United States in 2008-2012. Neuro Oncol 17 (Suppl 4): iv1-iv62, 2015.

2. Lassman AB and Holland EC: Glioblastoma multiforme - past, present and future. US Oncol Rev 1: 109-111, 2005.

3. Walker MD, Green SB, Byar DP, Alexander E Jr, Batzdorf U, Brooks WH, Hunt WE, MacCarty CS, Mahaley MS Jr, Mealey $\mathrm{J} \mathrm{Jr}$, et al: Randomized comparisons of radiotherapy and nitrosoureas for the treatment of malignant glioma after surgery. N Engl J Med 303: 1323-1329, 1980.

4. Keime-Guibert F, Chinot O, Taillandier L, Cartalat-Carel S, Frenay M, Kantor G, Guillamo JS, Jadaud E, Colin P, Bondiau PY, et al; Association of French-Speaking Neuro-Oncologists: Radiotherapy for glioblastoma in the elderly. N Engl J Med 356: $1527-1535,2007$

5. Leder K, Pitter K, Laplant Q, Hambardzumyan D, Ross BD, Chan TA, Holland EC and Michor F: Mathematical modeling of PDGF-driven glioblastoma reveals optimized radiation dosing schedules. Cell 156: 603-616, 2014.

6. Corwin D, Holdsworth C, Rockne RC, Trister AD, Mrugala MM, Rockhill JK, Stewart RD, Phillips M and Swanson KR: Toward patient-specific, biologically optimized radiation therapy plans for the treatment of glioblastoma. PLoS One 8: e79115, 2013.

7. Diffuse Low-Grade Gliomas in Adults: Natural History, Interaction with the Brain and New Individualized Therapeutic Strategies. Duffau H (ed). Springer, p2, 2013.

8. Stewart RD and Li XA: BGRT: Biologically guided radiation therapy-the future is fast approaching! Med Phys 34: 3739-3751, 2007.

9. Rockne R, Rockhill JK, Mrugala M, Spence AM, Kalet I, Hendrickson K, Lai A, Cloughesy T, Alvord EC Jr and Swanson KR: Predicting the efficacy of radiotherapy in individual glioblastoma patients in vivo: A mathematical modeling approach. Phys Med Biol 55: 3271-3285, 2010.

10. Rockne RC, Trister AD, Jacobs J, Hawkins-Daarud AJ, Neal ML, Hendrickson K, Mrugala MM, Rockhill JK, Kinahan P, Krohn KA, et al: A patient-specific computational model of hypoxia-modulated radiation resistance in glioblastoma using 18F-FMISO-PET. J R Soc Interface 12: 20150927, 2015.

11. Murray JD: Mathematical Biology. II Spatial Models and Biomedical Applications. Growth and Control of Brain tumors. Chapter 11, pp536-613. Springer, New York, 2003.

12. Harpold HL, Alvord EC Jr and Swanson KR: The evolution of mathematical modeling of glioma proliferation and invasion. $\mathrm{J}$ Neuropathol Exp Neurol 66: 1-9, 2007.

13. Swanson KR, Rostomily RC and Alvord EC Jr: A mathematical modelling tool for predicting survival of individual patients following resection of glioblastoma: A proof of principle. Br J Cancer 98: 113-119, 2008.
14. Tracqui P, Cruywagen GC, Woodward DE, Bartoo GT, Murray JD and Alvord EC Jr: A mathematical model of glioma growth: The effect of chemotherapy on spatio-temporal growth. Cell Prolif 28: 17-31, 1995.

15. Woodward DE, Cook J, Tracqui P, Cruywagen GC, Murray JD and Alvord EC Jr: A mathematical model of glioma growth: The effect of extent of surgical resection. Cell Prolif 29: 269-288, 1996.

16. Rockne R, Alvord EC Jr, Rockhill JK and Swanson KR: A mathematical model for brain tumor response to radiation therapy. $\mathrm{J}$ Math Biol 58: 561-578, 2009.

17. Jbabdi S, Mandonnet E, Duffau H, Capelle L, Swanson KR, Pélégrini-Issac M, Guillevin R and Benali H: Simulation of anisotropic growth of low-grade gliomas using diffusion tensor imaging. Magn Reson Med 54: 616-624, 2005.

18. Unkelbach J, Menze BH, Konukoglu E, Dittmann F, Le M, Ayache N and Shih HA: Radiotherapy planning for glioblastoma based on a tumor growth model: improving target volume delineation. Phys Med Biol 59: 747-770, 2014.

19. Herculano-Houzel S and Lent R: Isotropic fractionator: A simple, rapid method for the quantification of total cell and neuron numbers in the brain. J Neurosci 25: 2518-2521, 2005

20. Wang CH, Rockhill JK, Mrugala M, Peacock DL, Lai A, Jusenius K, Wardlaw JM, Cloughesy T, Spence AM, Rockne R, et al: Prognostic significance of growth kinetics in newly diagnosed glioblastomas revealed by combining serial imaging with a novel biomathematical model. Cancer Res 69: 9133-9140, 2009.

21. Barazzuol L, Burnet NG, Jena R, Jones B, Jefferies SJ and Kirkby NF: A mathematical model of brain tumour response to radiotherapy and chemotherapy considering radiobiological aspects. J Theor Biol 262: 553-565, 2010.

22. Gupta T and Dinshaw K: Modified optimal fractionation for poor prognosis malignant gliomas: An elusive search. Acta Oncol 44: 105-113, 2005.

23. Swanson KR, Alvord EC Jr and Murray JD: A quantitative model for differential motility of gliomas in grey and white matter. Cell Prolif 33: 317-329, 2000.

24. Beliën AT, Paganetti PA and Schwab ME: Membrane-type 1 matrix metalloprotease (MT1-MMP) enables invasive migration of glioma cells in central nervous system white matter. J Cell Biol 144: 373-384, 1999.

25. Giese A and Westphal M: Glioma invasion in the central nervous system. Neurosurgery 39: 235-250, discussion 250-252, 1996

26. Giese A, Bjerkvig R, Berens ME and Westphal M: Cost of migration: Invasion of malignant gliomas and implications for treatment. J Clin Oncol 21: 1624-1636, 2003.

27. Pedersen PH, Edvardsen K, Garcia-Cabrera I, Mahesparan R, Thorsen J, Mathisen B, Rosenblum ML and Bjerkvig R: Migratory patterns of lac-z transfected human glioma cells in the rat brain. Int J Cancer 62: 767-771, 1995.

28. Yoshida D, Watanabe K, Noha M, Takahashi H, Teramoto A and Sugisaki Y: Tracking cell invasion of human glioma cells and suppression by anti-matrix metalloproteinase agent in rodent brain-slice model. Brain Tumor Pathol 19: 69-76, 2002.

29. McAleese JJ, Stenning SP, Ashley S, Traish D, Hines F, Sardell S, Guerrero D and Brada M: Hypofractionated radiotherapy for poor prognosis malignant glioma: Matched pair survival analysis with MRC controls. Radiother Oncol 67: 177-182, 2003.

30. Hegi ME and Stupp R: Withholding temozolomide in glioblastoma patients with unmethylated MGMT promoter - still a dilemma? Neuro Oncol 17: 1425-1427, 2015.

31. Kiley F, Twomey F: Butterfly glioma involving splenium of corpus callosum. Int J Clin Med Imaging 2: 1000277, 2015. 